Lepr Rev (1994) 65, 81-87

\title{
Editorial
}

\section{SOME POINTS ON THE ELIMINATION OF LEPROSY}

During the last decade, and on a large scale since 1987-88, the widespread use of multidrug therapy (MDT) regimens in leprosy control programmes, as recommended by WHO in $1982^{5}$ and in $1988^{6}$, has dramatically changed the leprosy situation. ${ }^{9}$ While in 19865.3 million leprosy cases were registered worldwide, there are now 2.3 million patients registered for treatment, of whom 1,100,000 are on MDT. Since the introduction of MDT regimens, 4.3 million leprosy patients have been treated with this method and consequently classified as 'cured', with or without sequelae (with the exception of a negligible number of relapses).

However, it is recognized that, although in many countries MDT coverage is progressing, in others where operational problems exist, MDT coverage may not indrease as expected. In general, the reason for such a decline in MDT expansion is that, until recently, the method has been implemented in countries or areas which have adequate infrastructure and facilities, and now it is being extended to 'difficult areas' with poor working conditions.

It was at this juncture that in May 1991 the World Health Assembly adopted a Resolution $^{8}$ to eliminate leprosy as a public health problem by the year 2000 , and defined elimination as corresponding to a level of prevalence below 1 per 10,000 population.

This resolution has raised a series of questions and concerns. ${ }^{2}$ It has also stimulated a great deal of discussion, both in WHO and in other quarters, in order to clarify a number of issues and to develop the appropriate approach for attaining the elimination of leprosy. WHO, through several consultations and meetings at various levels, ${ }^{7}$ evolved a strategy for eliminating leprosy as a public health problem at country level.

\section{The current leprosy situation}

Based on WHO information, the current leprosy situation in the world is summarized in Table 1.

The analysis of the distribution of leprosy by country shows that, if leprosy remains a public health problem in 87 countries or areas, 25 countries have almost $95 \%$ of the leprosy burden in the world. Table 2 shows the registered prevalence and MDT coverage for these 25 most affected countries. ${ }^{9}$ 
Table 1. Summary of the global leprosy situation

Estimated cases

Registered cases

Registered cases on MDT

New cases detected in 1992

Cumulative number of patients having completed MDT

Population living in countries with prevalence above 1 per 10,000

People with visible disabilities resulting from leprosy

Expectations:

Patients to be treated between 1993 and 2000:

Additional cost involved in elimination strategy

$3,055,210$
$2,291,581$
$1,117,508$
653,354
$4,237,712$
$2,400,000,000$
$2-3$ million
6-7 million
US\$ 420 million
to US\$ 600 million

\section{The current strategy for leprosy control and the elimination strategy}

\section{CURRENT STRATEGY}

The current strategy for leprosy control is based upon: (a) early identification of cases and standardized classification; (b) appropriate treatment of the same with WHO-MDT regimens; and, (c) health education directed to patients and their communities. The result

Table 2. Magnitude of the leprosy problem in the top 25 endemic countries

\begin{tabular}{|c|c|c|c|c|c|c|c|}
\hline Countries & Year* & $\begin{array}{l}\text { Registered } \\
\text { Cases }\end{array}$ & $\begin{array}{c}\text { Prevalence } \dagger \\
\text { per } 10,000\end{array}$ & $\begin{array}{l}\text { Patients } \\
\text { on MDT }\end{array}$ & $\begin{array}{c}\text { MDT } \\
\text { Coverage \% }\end{array}$ & $\begin{array}{l}\text { Completed } \\
\text { MDT }\end{array}$ & $\begin{array}{l}\text { Cumulative } \\
\text { MDT cov. } \%\end{array}$ \\
\hline India & 92 & 1459338 & $16 \cdot 40$ & 742988 & $50 \cdot 91$ & 3500000 & $85 \cdot 56$ \\
\hline Brazil & 91 & 250066 & 16.00 & 62041 & $24 \cdot 81$ & 23008 & $31 \cdot 15$ \\
\hline Indonesia & 92 & 74683 & 3.90 & 36907 & $49 \cdot 42$ & 74113 & $74 \cdot 61$ \\
\hline Bangladesh & 92 & 19932 & 1.63 & 13007 & $65 \cdot 26$ & 35618 & $87 \cdot 53$ \\
\hline Myanmar & 92 & 57389 & $11 \cdot 19$ & 31900 & $55 \cdot 59$ & 96181 & $83 \cdot 40$ \\
\hline Nigeria & 92 & 62080 & $7 \cdot 01$ & 15088 & $24 \cdot 30$ & 2251 & 26.95 \\
\hline Sudan & 92 & 31028 & $11 \cdot 62$ & 7198 & $23 \cdot 20$ & 2113 & $28 \cdot 10$ \\
\hline Philippines & 92 & 14925 & $2 \cdot 28$ & 9506 & 63.69 & 28491 & $87 \cdot 52$ \\
\hline Iran & 91 & 10487 & 1.84 & 10487 & $100 \cdot 00$ & 0 & $100 \cdot 00$ \\
\hline Vietnam & 91 & 18342 & 2.63 & 11004 & 59.99 & 23284 & $82 \cdot 37$ \\
\hline Madagascar & 92 & 5290 & $4 \cdot 13$ & 5188 & 98.07 & 8037 & $99 \cdot 23$ \\
\hline Egypt & 91 & 8696 & $1 \cdot 59$ & 8696 & $100 \cdot 00$ & 8868 & $100 \cdot 00$ \\
\hline Nepal & 92 & 22812 & $11 \cdot 37$ & 15318 & $67 \cdot 15$ & 22735 & 83.55 \\
\hline China & 92 & 20003 & 0.17 & 19744 & $98 \cdot 71$ & 47073 & $99 \cdot 61$ \\
\hline Zaire & 92 & 7736 & $2 \cdot 04$ & 2181 & $28 \cdot 19$ & 41957 & $88 \cdot 82$ \\
\hline Mozambique & 92 & 19216 & $11 \cdot 63$ & 2360 & $12 \cdot 28$ & 533 & $14 \cdot 65$ \\
\hline Colombia & 89 & 18983 & $5 \cdot 54$ & 7229 & 38.08 & 1668 & 43.08 \\
\hline Mexico & 92 & 16732 & 1.81 & 7372 & $44 \cdot 06$ & 1322 & $48 \cdot 16$ \\
\hline Ethiopia & 91 & 12041 & $2 \cdot 31$ & 11680 & $97 \cdot 00$ & 42938 & $99 \cdot 34$ \\
\hline Guinea & 92 & 6942 & $11 \cdot 36$ & 6942 & $100 \cdot 00$ & 18366 & $100 \cdot 00$ \\
\hline Cote d'Ivoire & 92 & 6483 & $5 \cdot 01$ & 4696 & $72 \cdot 44$ & 11910 & $90 \cdot 28$ \\
\hline Mali & 92 & 12710 & 12.95 & 3114 & $24 \cdot 50$ & 3279 & 39.98 \\
\hline Chad & 92 & 6952 & $11 \cdot 64$ & 761 & 10.95 & 2472 & $34 \cdot 31$ \\
\hline Niger & 92 & 6468 & $7 \cdot 84$ & 447 & 6.91 & 1087 & $20 \cdot 30$ \\
\hline Pakistan & 90 & 9611 & 0.74 & 2389 & $24 \cdot 86$ & 6174 & $54 \cdot 25$ \\
\hline Total & & 2178945 & 6.45 & 1038243 & $47 \cdot 65$ & 4003478 & $81 \cdot 55$ \\
\hline
\end{tabular}

* Last year from which the data are available.

† Using the 1992 mid-year population data, from World Population Prospects 1990 (UN).

From Weekly Epidemiological Record, 1993, 68: 181-188. WHO June 1993. 
of this strategy has been rapid, drastic reductions in prevalence; decreases by $90 \%$ within 5 years are common in well-organized control programmes. ${ }^{3}$ However, parallel decreases of incidence are not observed although, in a few instances, a relative decrease in incidence has been attributed to MDT. Despite the fact that there is very little proof of the impact of MDT on the transmission of Mycobacterium leprae, it is hoped that the decrease in prevalence will, by reducing the sources of infection, ultimately result in a fall of incidence. $^{4}$

In view of the contradictory results obtained in several prospective studies on the preventive value of BCG against leprosy, WHO does not recommend BCG vaccination as part of its strategy for leprosy control. However, recent case control studies may lead to a revision of this opinion. A recent prospective study in Venezuela in which a mixture of BCG and killed $M$. leprae was used as a candidate vaccine has given inconclusive results. Results of other vaccine trials using $M$. leprae mixed with cultivable mycobacteria other than BCG are expected to be available after $1995 .^{10}$

\section{ELIMINATION STRATEGY}

The elimination strategy includes exactly the same technical components as that of leprosy control but it is different in two aspects: a time limit (the year 2000) has been added and a target (prevalence lower than 1 per 10,000) has been ascribed. In other words, the elimination strategy is just a natural expansion and strengthening of leprosy control based on MDT. If no time limit had been fixed, the step designated as elimination would have been considered as a target to be reached at any time by each individual country.

The additional constraints of 'time limit' and 'prevalence target' will, in certain areas, make it difficult for the elimination strategy to be implemented: all patients, including those difficult to reach, those reluctant to be diagnosed as having leprosy, those who do not care - not to mention those with problems of differential diagnosis-will have to be localized and then persuaded to accept a full course of an appropriate MDT regimen.

The WHO Leprosy Unit has worked out a model elimination strategy with successive phases, each of which have specific activities. ${ }^{4}$ As part of this process, several important issues were clarified. For instance, because of the well-known uneven geographical distribution of leprosy, it was necessary to decide on a level of population for use as a denominator to monitor the decrease in prevalence and incidence rates. After discussion, it was found that, for practical reasons, elimination should be declared first at national level and, later on, at the level of regions or districts which originally had high prevalence.

\section{Improvements to be expected from the elimination strategy}

It appears that the elimination strategy could render leprosy control activities more effective in several respects, as summarized below.

\section{IMPROVED CASE DETECTION}

It is well known that, as a rule, MDT regimens attract patients much more than did the previous dapsone monotherapy. However, when caref ul estimates are made of the total number of existing cases in MDT-based control programmes, it is generally concluded 
that the actual number of patients is significantly greater than the number of registered cases. ${ }^{9}$ The identification of non-registered patients is obviously an essential step in the expansion of the MDT coverage if we are to reduce the gap between registered and estimated cases.

\section{IMPROVED MONITORING OF LOW-INCIDENCE SITUATIONS}

In a proportion of endemic countries the control strategy has been followed by very low prevalence and case detection levels. With the elimination strategy, prevalence and case detection rates will be further reduced to minimal levels. In the absence of a reliable test for subclinical infection, which could make the monitoring of minimal incidence situations possible, the elimination strategy has stimulated an effort to develop methods for monitoring such situations. So far, it is expected that these methods will include a set of selected indicators and, at the ultimate phase of the elimination process, the use of sentinel centres. ${ }^{1}$

There is one stage in the elimination process at country level which deserves special consideration. It is when the prevalence rate in its decreasing process becomes equal to the case detection rate (or when the prevalence rate becomes lower than the case detection rate, as a result of PB patients diagnosed during the first half of the year and having completed MDT by the end of the same year). This stage is most interesting; when it has been reached, in subsequent years, if MDT has an impact on incidence, taking into account cases infected before the introduction of MDT, successive annual 'incidence' rates should be decreasing.

\section{IMPROVED MDT COVERAGE}

For many reasons MDT-delivery to patients can be difficult, resulting in poor compliance of the patient. It is clearly indicated in the elimination strategy ${ }^{4}$ that flexibility is important if we are to facilitate patients' accessibility to MDT. Adaptation to patients' constraints, in terms of time and a place for drug supply, is an obvious and simple possibility. In the more distant future, some of the new drug regimens currently under evaluation, e.g. regimens with monthly supervised intake of all drugs together and no daily unsupervised intake, could be a solution in certain circumstances.

\section{OPPORTUNITY TO GO AS FAR AS POSSIBLE IN LEPROSY CONTROL}

Improved case detection, improved monitoring of low incidence situations, and improved MDT coverage are the main technical elements of improved leprosy control. However, I strongly feel that those responsible for leprosy work should not only try to increase the impact of the available technology on the leprosy problem, but should do so within the minimum time interval.

Thus, the responsible authorities should take advantage of the ongoing control strategy to build the elimination strategy on the already existing infrastructure and facilities, this being obviously the most cost-effective option. However, there are concerns which should be taken into account, the two most important of which are, perhaps, the following:

(a) For all those involved in leprosy activities, it is of course very gratifying to see that the problem is being alleviated through MDT-based control. However, there is the 
possibility that, in some programmes, the authorities might be satisfied with a reduction in prevalence of say $90 \%$ of already registered cases, and therefore may not be inclined to make special efforts to chase non-registered cases, nor to reduce the prevalence to 1 per 10,000 .

(b) As a consequence of the drastic reduction in prevalence resulting from the control strategy, the donor agencies have expressed concern that fund-raising for leprosy activities could become difficult now that the size of the leprosy problem has so much decreased. $^{2}$

In fact, at this very moment, all partners involved, especially leprosy services and donor agencies, should not relax their efforts; on the contrary, they should redouble their activities so as to achieve, as soon as possible, the maximum that can be achieved for leprosy sufferers using the existing tools.

\section{Problems inherent to the elimination strategy}

NEED FOR ADDITIONAL RESOURCES

WHO has made an estimate of the additional financial resources required to implement the elimination strategy. This estimate is from US\$ 420 to US\$ 600 million for the period 1993 to 2000. Although this amount is substantial, it is not beyond the possibilities of donor agencies.

\section{NEED TO GIVE PRIORITY TO DISABILITY PREVENTION AND MANAGEMENT}

Early diagnosis and appropriate MDT are the required basis for the prevention of disabilities. Other more specific activities aimed at disability prevention and management, such as early detection and treatment of nerve damage, education of patients on simple measures for protecting eyes, hands and feet, reconstructive surgery, etc., are also quite important.

Some MDT-based control programmes have taken advantage of the reduced number of patients by incorporating into their activities those related to prevention and management of disabilities. If this is not done at an early stage, these activities should be integrated into the elimination programme during its phase of consolidation.

\section{Conclusion}

To conclude, I have 3 important questions to ask.

IS THE ELIMINATION STRATEGY AN IMPROVEMENT COMPARED TO THE CONTROL STRATEGY?

As discussed above, it would appear that, if compared to the control strategy, the elimination strategy is likely to result in increased case detection, increased MDT coverage and better monitoring of progress.

The reasons for which the elimination strategy should be more effective than the control strategy are its specific components: a 'time limit' and a 'defined target'. 
WHAT IS THE IMPORTANCE OF THE DRAWBACKS OF THE ELIMINATION STRATEGY?

(a) The need for additional resources does not appear a serious constraint.

(b) There will be important operational difficulties:

(1) related to activities (case detection, MDT delivery, treatment compliance, etc. ); and

(2) related to monitoring of progress as we do not have an adequate indicator for measuring progress in low prevalence/incidence situations.

\section{IS IT TIMELY TO ENGAGE IN AN ELIMINATION STRATEGY?}

It seems that if the elimination strategy is implemented as a further step and natural expansion of the control strategy, there can be only advantages, such as:

(a) It can be built on the inf rastructure and facilities already established for the control strategy, and can take advantage of the experience gained by its implementation.

(b) In terms of caseload, a better operational and possibly epidemiological (concerning incidence) impact than with the control strategy; in any case, the greatest impact which could be obtained from an MDT-based strategy.

It is important to note that these advantages will become effective only if the two following conditions are met.

(a) Operational methods are developed to overcome operational difficulties. This should be feasible, although unconventional solutions to some problems should be accepted.

(b) Methods for monitoring progress in low prevalence/incidence situations will have to be developed. This may cause real difficulties. However, full use should be made of the ratio prevalence/case detection rate. This indicator may prove to have a greater significance than expected.

Altogether, it seems that the advantages of implementing the elimination strategy in sequence with the control strategy, and as a natural expansion of the latter, outweighs the disadvantages. In contrast, if implementing the elimination strategy was delayed, there is no certainty that the methods required to facilitate case-detection and case-holding, and to monitor progress in low prevalence/incidence situations, would be developed without the commitment of national authorities to eliminate leprosy as a public health problem.

\section{St Armou}

H. SANSARRICQ

64160 Morlaas

France

\section{References}

1 Daumerie D, Réseau de surveillance épidémiologique et évaluation des progrès vers l'élimination. Réunion des responsables de la lutte antilépreuse, Yaounde, 1993.

2 Fine, PEM. Reflections on the elimination of leprosy. (Editorial) Int. J. Lepr. 1991; 60: 71-80.

3 Noordeen SK. Elimination of leprosy as a public health problem. (Editorial) Lepr Rev, 1992; 63: 1-4.

4 Noordeen SK. Global strategy for the elimination of leprosy as a public health problem. Personal communication.

5 WHO Study Group on Chemotherapy of Leprosy for Control Programmes, Geneva: World Health Organization, 1982, Technical Report Series 675. 
${ }^{6}$ WHO Expert Committee on Leprosy. Sixth Report, Geneva. World Health Organization, 1988. Technical Report Series, 768.

7 World Health Organization. Report on the Second meeting of the WHO Working Group on Leprosy Control, Geneva: World Health Organization, 1992. WHO.

8 Resolution (WHA44.9), May 1991.

9 Progress with implementation of MDT. Weekly Epidemiological Record, No. 21, May 1993.

10 Report of the Workshop on approaches to epidemiology, prevention and control, 14th International Leprosy Congress, Orlando, 1993. 